\title{
Estimation of Rational Modes and Regimes of Production of Sod Peat with Milling-Forming Machine by Quality Indicators
}

\author{
Anna Guseva ${ }^{1, *}$ \\ ${ }^{1}$ Tver State Technical University (TvSTU), 170026, Afanasy Nikitin emb., 22, Tver, Russia
}

\begin{abstract}
Modern trends in designing mining machines dictate that their power consumption should be reduced. However, the end product quality is often not taken into account. The objective of the research is to improve the operation of a screw press of the milling and moulding machine used for producing sod peat in order to ensure the necessary quality of domestic fuel. To that end, the authors conducted an experiment simulating the sod peat production and determined the density and strength of the sod obtained during the improved machine operation. The experiments were carried out for the three pressure coefficients, further, the coefficient influence on the sod density and strength was evaluated. The sod peat density was assessed with modern electronic devices having a low error rate. The novelty of the research is to reconcile the issues of ensuring the minimum power consumption for producing domestic sod biofuel with its qualitative characteristics. The obtained indicators of the sod peat quality in terms of density and strength prove its improved moulding along with the minimum power consumption at a pressure coefficient of 2.22. Thus, the improved production of sod peat requires minimum power consumption, provides the required quality of the end sod and reduces the loss of feedstock due to crumbling.
\end{abstract}

\section{Introduction}

The production of sod domestic fuel solves the problem of energy autonomy and safety of settlements in the absence of local gas supply and the presence of abundant peat deposits throughout the world $[1,2]$.

The main focus of designing peat extraction machines and equipment is on reducing the power consumption of production processes, increasing the equipment mobility, and, consequently, reducing operating costs. However, the equipment design features in relation to the end product quality are not taken into account. The most power-consuming operation

\footnotetext{
* Corresponding author: guseva ann@,mail.ru
} 
in the sod peat production technology is slot-milling and moulding, which account for about $50 \%$ of all operating costs [3].

The research relevance is justified by the need to reduce the production cost of sod peat and the search for improved milling machine parameters to reduce power consumption and to maintain the required quality characteristics of the end fuel. Sod peat is a fairly light fuel with a calorific value of $3200 \mathrm{Kcal} / \mathrm{kg}[3,4]$. The required calorific value is provided with a sod reaching a certain density after moulding and drying. Technological operations to produce and transport domestic sod peat fuel are performed in terms of the fact that an important indicator of the fuel safety is its bending strength.

The quality of domestic peat sod fuel is ensured by the required calorific value and the retention of the sod mouthpiece shape.

The required calorific value is ensured by the sod peat density at the conditioned moisture stage in the absence of microdefects and cracks after moulding and drying. The mouthpiece shape retention is determined by the bending strength making possible subsequent technological operations to produce sod peat and the absence of crumbling. The sod peat strength, like no other characteristic, gives an idea of peat quality and safety during production and transportation.

The sod peat density determines the sod peat calorific value at the conditioned moisture stage in the absence of microdefects and cracks after moulding and drying. The density should be $750-1100 \mathrm{~kg} / \mathrm{m}^{3}$. The bending strength of sod peat should be at least $3.5-4.5 \mathrm{MPa}$ [3-6]. Ensuring such sod peat density and strength as well as minimizing the process power consumption will allow the domestic fuel production process to be improved.

In case these quality indicators are provided with improved parameters and modes of sod peat production by a milling and moulding machine, the goals to reduce the operating costs of domestic sod fuel production will be achieved with reaching the required quality indicators.

\section{Review}

The laboratory equipment of the Department of Technological Machines and Equipment of Tver State Technical University, Russia, helps conduct experimental peat dispersion and moulding, which was carried out with a screw press having 5 speed modes, 3 replaceable mouthpieces and 2 screws with different pitches (Fig. 1). The feedstock moisture content, the useful capacity, the press performance, and the process power consumption were determined in the course of the research $[3,5]$.

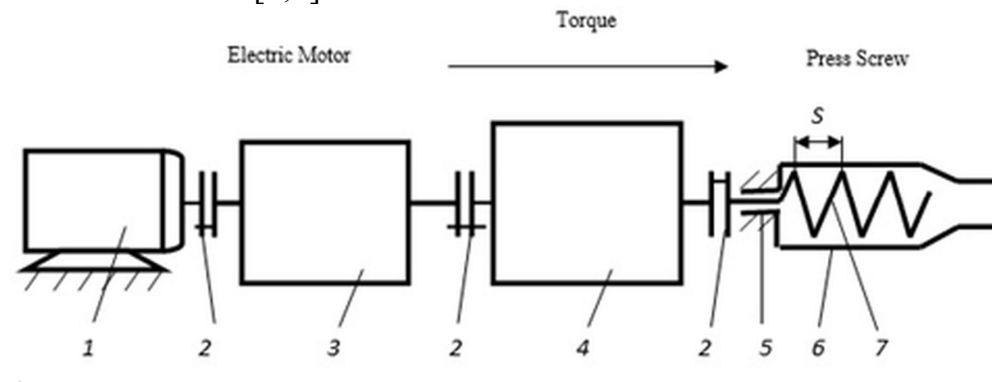

Fig. 1. Labware Arrangement: 1 - an electric motor; 2 - a coupling; 3 - a gearbox; 4 - a cylindrical reducer; 5 - screw support bearings; 6 - a pressure pipe (barrel casing); 7 - a screw; 8 - a cylindrical mouthpiece.

The experiments showed that the two screws having 110 and $50 \mathrm{~mm}$ pitch and installed by turns in the screw press gave the lowest moulding power consumption corresponding to 
the screw rotation frequency of 220-320 rpm when the screw worked with a $50 \mathrm{~mm}$ pitch, i.e. equal to half of the outer diameter of the screw. The higher density of the moulded sods corresponded to the higher rotation frequency and the smaller screw pitch [1]. Thus, the improved parameters and operating modes of a moulding unit were obtained. However, the use of three mouthpieces with different internal diameters required that the effect of the diameter on sod peat density and strength was identified when the screw press operated in the minimum power consumption mode [3,5-6]. After shadow drying, the density of the sods obtained in different moulding modes was determined (Fig. 2). Then, the sod peat was tested on a stand creating a destructive load [7]. These experiments were carried out with modern digital measuring equipment. In general, higher results were obtained due to manual control of the technological process and a soft drying mode without sharp changes in day and night temperatures and air moisture indexes.

In order to use the method of geometric similarity and the possibility of controlling power consumption in producing sod peat, $[3,5]$ introduce the concept of 'pressure coefficient' which describes the degree of peat compaction in the pressure part of a screw press pipe and a mouthpiece:

where $D$ is the inner diameter of the pressure pipe; $d_{o u}$ is the outer diameter of the pipe being the base for the screw spiral; $d$ is the mouthpiece diameter; $m$ is the number of mouthpieces.

The parameters of the laboratory screw press used in the research are $D=102 \mathrm{~mm}, d_{o u}=$ $49 \mathrm{~mm}$. Thus, the pressure coefficients $k_{p}$ for mouthpieces with a diameter of 52, 60, and 92 $\mathrm{mm}$ are $2.96,2.22$, and 0.95 respectively.

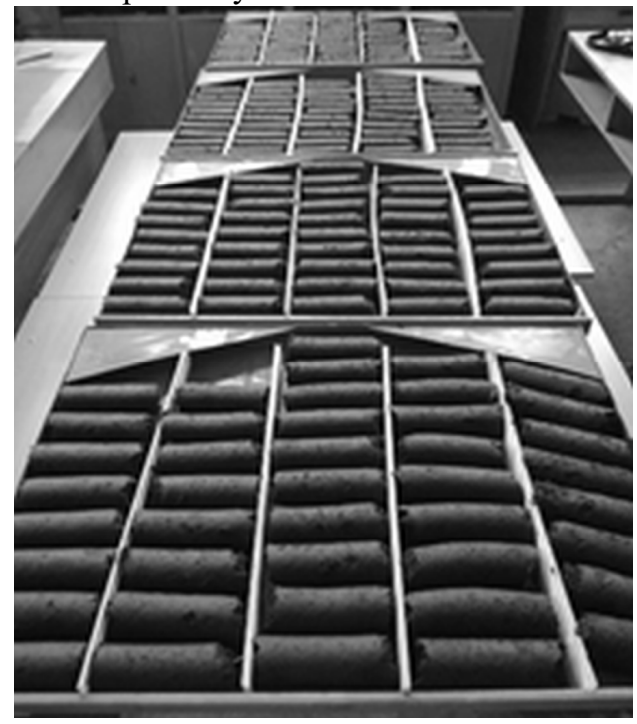

Fig.2. Shadow drying of sod peat.

The class mark of the power consumption minimum was described with the cross-plots of the dependence of the sod peat density and strength at the conditioned moisture on the moulding power consumption at different screw press parameters (Fig. 3). 


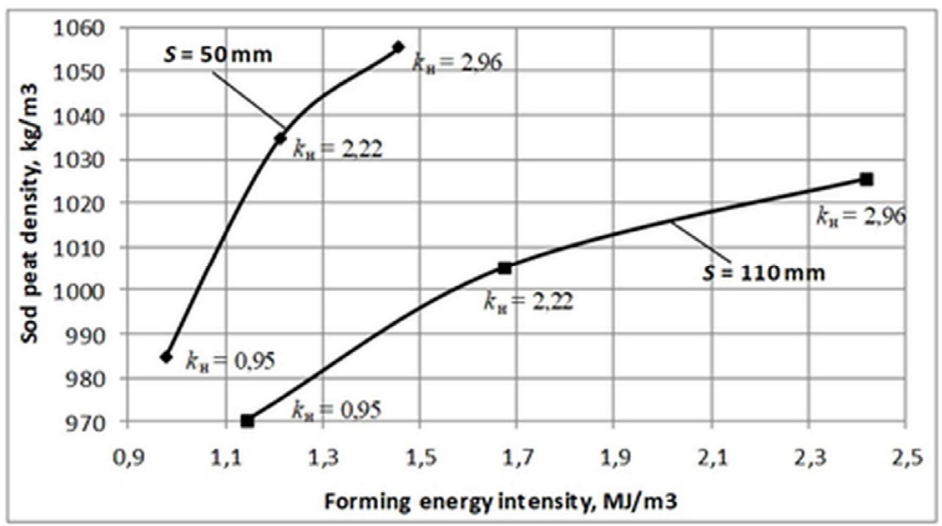

Fig. 3. Sod peat density dependence of energy intensity at a rotation speed of screw $n=270$ RPM.

According to the obtained dependences, the peat density growth decreases markedly when the $k_{p}$ changes from 2.22 to 2.96 . At the same time even at $k_{p}=2.22$, the density reaches the conditioned values. Therefore, the conclusion was made that moulding with a pressure coefficient $k_{p}=2.22$ can be considered improved.

The class mark of the power consumption minimum was also described with the plots of the dependence of the sod peat strength at the conditioned moisture on the moulding power consumption at different screw press parameters (Fig. 4).

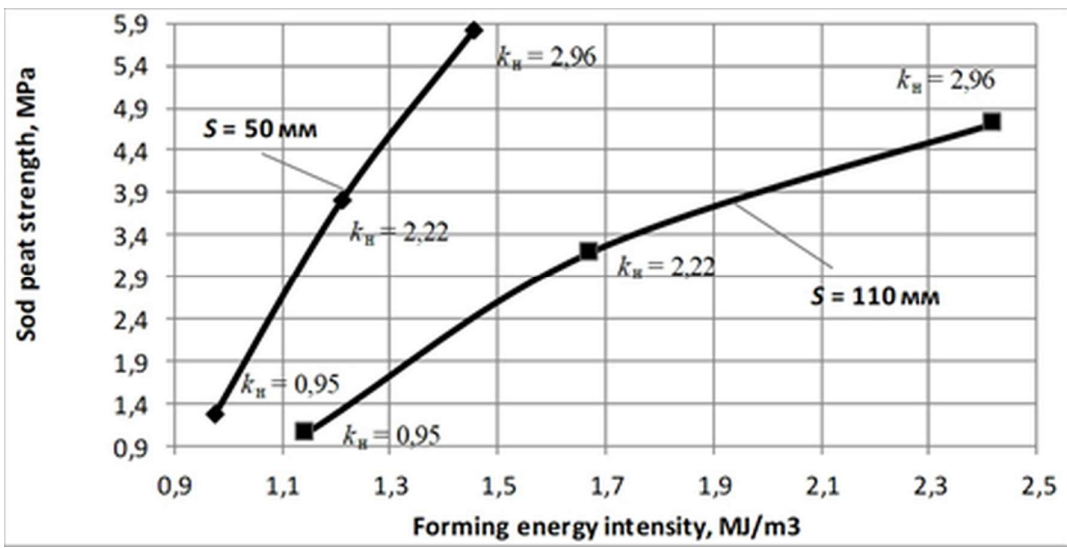

Fig. 4. Sod peat strength dependence of energy intensity at a rotation speed of screw $n=270$ RPM.

The results presented are consistent with the results obtained for the density. The peat strength growth significantly decreases with a $k_{p}$ change from 2.22 to 2.96. At the same time, even at $k_{p}=2.22$, the density and strength reach the conditioned values. Therefore, the authors conclude that moulding with a pressure coefficient $k_{p}=2.22$ can be considered as improved.

Thus, the required quality of the end domestic peat fuel, along with the minimum power consumption of the production process, is possible when moulding is done with a pressure coefficient $k_{p}=2.22$. In addition, the improved sod peat development in terms of the end product quality at the conditioned moisture stage is moulding with a screw rotation frequency $n=220-320 \mathrm{rpm}$ and a screw pitch equal to half of its diameter $S=0.5 D$. 


\section{Conclusion}

In the modern world, the search for domestic fuels alternative to gas is an urgent task especially for remote areas including the Far North. Abundant peat deposits can attract our attention as an energy source. Improved sod peat production, along with the necessary quality of end energy products, opens up positive prospects for the domestic sod peat production [812]. All the experiments carried out show that the growth of sod peat density and strength in moulding at a rotation frequency of $270 \mathrm{rpm}$ decreases when the pressure coefficient changes from 2.22 to 2.96. Therefore, the improved sod peat production, in terms of the end product density and strength at the stage of conditioned moisture, proves to be moulding at a pressure coefficient of 2.22 .

The obtained values of sod peat density and strength are slightly higher than the values obtained in the field due to the manual control of extraction and soft drying.

Along with the improved values of the screw rotation frequency $n=220-320 \mathrm{rpm}$ and the screw pitch $S=0.5 D$, the pressure coefficient of 2.22 is an improved performance parameter of the screw press of a milling and moulding machine to produce sod peat.

The improved design and operation of milling and moulding machines will reduce the specific power consumption in production and provide the required fuel quality in terms of density and strength as well as reduce the peat loss during extraction. Thus, the power supply of remote areas with a high-quality product can be provided with the on-site improved production of domestic peat biofuel.

\section{References}

1. V. Panov, O. Misnikov, Mining Journal, 7, 108-112, (2015)

2. O. Misnikov, Mires and Peat, 18(22), 1-15, (2016)

3. A. Yablonev, A. Guseva, Mining informational and analytical bulletin, 2, 1, (2019)

4. V. Belyakov, A. Kuporova, E3S Web of Conf. 174, 01008 (2020)

5. A. Yablonev, A. Guseva, E3S Web of Conf. 105, 01033 (2019)

6. A. Guseva, A. Yablonev, Mining informational and analytical bulletin, 3, 2 (2020)

7. A. Yablonev, A. Guseva, E3S Web of Conf. 105, 01033 (2020)

8. O. Misnikov, E3S Web of Conf. 41, 01046 (2018)

9. V. Belyakov, A. Kuporova, E3S Web of Conf. 41, 01047, (2018)

10. O. Misnikov, E3S Web of Conf. 105, 01010, (2019)

11. O. Misnikov, E3S Web of Conf. 174, 01024 (2020)

12. O. Misnikov, E3S Web of Conf. 21, 01020 (2017) 